\title{
Pore Volume and Porosity Changes under Uniaxial Strain Conditions
}

\author{
Robert W. Zimmerman ${ }^{1}$
}

Received: 22 March 2017 / Accepted: 23 June 2017 / Published online: 27 July 2017

(C) The Author(s) 2017. This article is an open access publication

\begin{abstract}
Expressions for the changes that occur in the pore volume and the porosity of a porous rock, due to changes in the pore pressure, overburden stress, and temperature, are derived within the context of the linearised theory of poroelasticity. The resulting expressions are compared to the commonly used equations proposed by Palmer and Mansoori, and it is shown that their expressions are consistent with the exact expressions if their factor $f$ is identified with $(1+v) / 3(1-v)$, where $v$ is the Poisson's ratio of the porous rock. Finally, the first derivation is given, within the context of the fully coupled linearised theory of poroelasticity, that under uniaxial strain, the partial differential equation that governs the evolution of the pore pressure is a pure diffusion equation, with a total compressibility term that (exactly) equals the sum of the fluid compressibility and the uniaxial pore volume compressibility.
\end{abstract}

Keywords Poroelasticity · Porosity · Uniaxial strain · Coalbed methane · Palmer-Mansoori equation

\section{List of symbols}

$B \quad$ Skempton's induced pore pressure coefficient, Eq. (28)

$C_{\mathrm{bc}} \quad$ Bulk compressibility with respect to confining pressure, Eq. (1) (1/Pa)

$C_{\mathrm{bp}} \quad$ Bulk compressibility with respect to pore pressure, Eq. (1) (1/Pa)

$C_{\mathrm{f}} \quad$ Compressibility of the pore fluid $(1 / \mathrm{Pa})$

$C_{\mathrm{m}} \quad$ Mineral phase compressibility $(1 / \mathrm{Pa})$

$C_{\mathrm{pc}} \quad$ Pore compressibility with respect to confining pressure, Eq. (2) $(1 / \mathrm{Pa})$

$C_{\mathrm{pp}} \quad$ Pore compressibility with respect to pore pressure, Eq. (2) (1/Pa)

$C_{\mathrm{pp}}^{\mathrm{uni}} \quad$ Uniaxial pore volume compressibility, Eq. (43) (1/Pa)

$凶 \quad$ Robert W. Zimmerman

r.w.zimmerman@ic.ac.uk; r.w.zimmerman@imperial.ac.uk

1 Department of Earth Science and Engineering, Imperial College, London SW7 2AZ, UK 


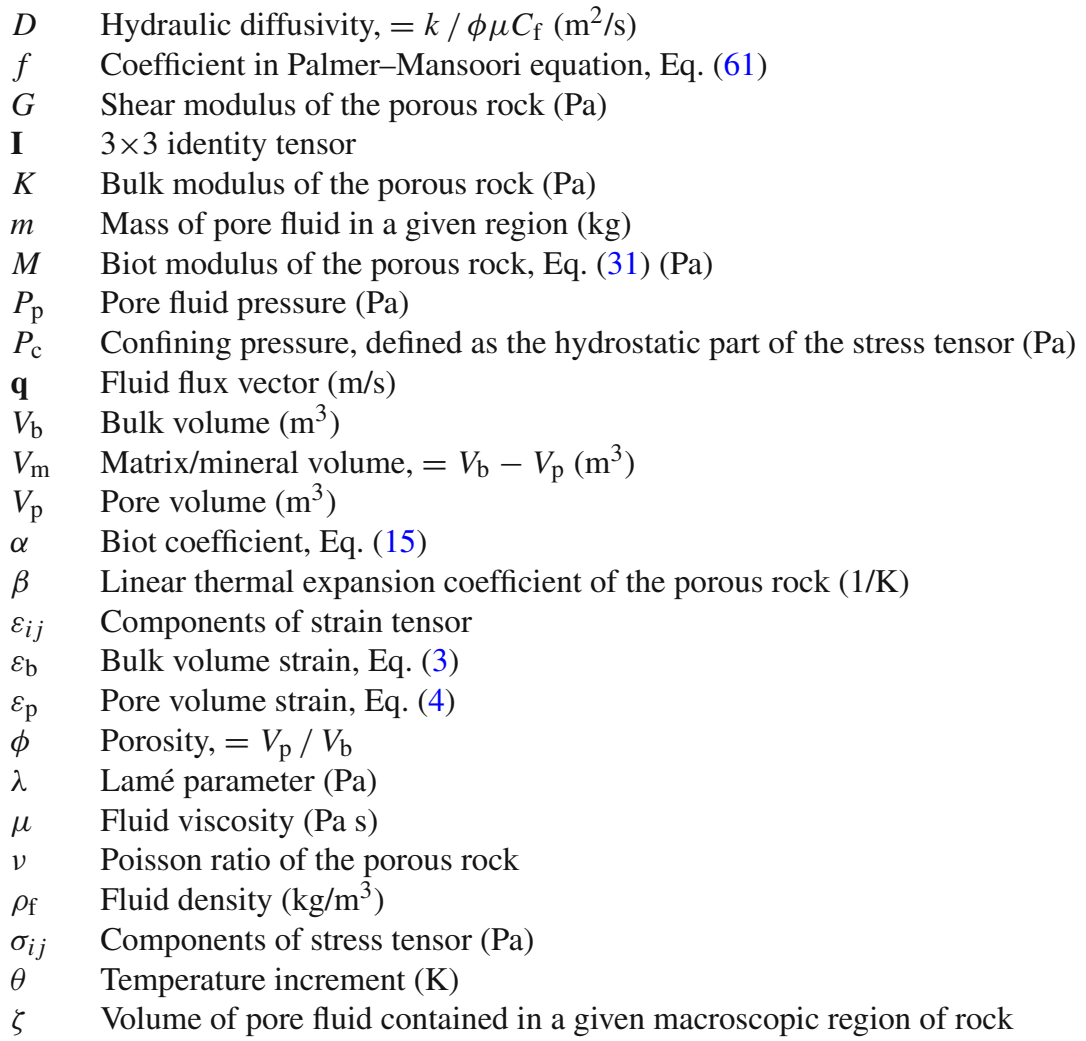

\section{Introduction}

When fluid is produced from a reservoir, it is generally assumed that the reservoir deforms under conditions of zero lateral strain (Fjaer et al. 2008). Under conditions of uniaxial vertical strain, changes in parameters such as the pore volume and the porosity will be functions of the changes in vertical overburden stress, pore pressure, and temperature. If these latter changes are suitably small, material parameters such as the elastic moduli and thermal expansion coefficient can be considered to be constant, and the changes in pore volume or porosity will be linearly related to the changes in overburden stress, pore pressure, and temperature, through some coupling coefficients. If these changes are not small, the resulting linearised relations can be considered to be incremental relations, that hold over a certain range, and the coupling coefficients can then be allowed to vary with stress and temperature, if necessary.

The coefficient that relates the changes in pore volume to the change in pore fluid pressure is required as an input for material balance calculations (Chierici 1994). The coefficient that relates the changes in porosity to the change in pore fluid pressure is required in models that relate the porosity changes to permeability changes (Seidle et al. 1992). This compressibility coefficient is also used in the pressure diffusion equation that forms the basis of well test analysis (Matthews and Russell 1967). Despite the importance of these uniaxial strain pore/porosity compressibility coefficients in reservoir engineering, they have not often been studied rigorously within the context of the coupled theory of poroelasticity. 
The purpose of the present paper is to derive expressions for these uniaxial strain pore/porosity compressibility coefficients, in terms of the more naturally defined (and more readily measured) parameters such as the Poisson ratio and the hydrostatic pore volume compressibility. Additionally, it will be shown that under conditions of uniaxial strain, the hydromechanically coupled partial differential equation that governs the pore pressure evolution reduces (exactly) to a pure diffusion equation, with an effective total compressibility that is equal to the sum of the fluid compressibility and the uniaxial pore volume compressibility. Although this latter result is perhaps not unexpected, it does not appear to have been previously proven. Furthermore, the present derivation will show that this result is exact, within the context of coupled poroelasticity.

\section{General Theory of Linearised Isotropic Poroelasticity}

The theory that accounts for coupled hydromechanical behaviour of fluid-saturated porous rocks is known as poroelasticity. This theory was originated independently by Biot (1941) and Frenkel (1944), and developed further by, among others, Verruijt (1969), Rice and Cleary (1976), and Detournay and Cheng (1993). Although numerous derivations of the equations of linearised poroelasticity have been given, the resulting theories are all essentially equivalent. The only possibly variance between the different formulations relates to whether or not the assumption is made that the solid phase is composed of a single material with locally homogenous properties. This commonly made assumption of micro-homogeneity (Zimmerman 1991), which Cheng (2016) refers to as the "ideal porous medium" assumption, leads to the simplest version of the theory of linearised poroelasticity and will be assumed throughout the following analysis.

The derivation given below of the governing equations of poroelasticity follows that of Zimmerman (2000a). This approach makes a strong connection with the pore volume and therefore is very convenient for the present purposes. Although the derivation given in this section will unavoidably repeat material that can already be found in the archival literature, the following concise but complete derivation will allow the subsequent results regarding processes that occur under conditions of uniaxial strain to be obtained in a self-contained manner that does not require frequent appeal to seemingly ad hoc external equations.

The starting point is the definition of the following four porous rock compressibilities (Zimmerman et al. 1986):

$$
\begin{aligned}
C_{\mathrm{bc}} & =\frac{-1}{V_{\mathrm{b}}}\left(\frac{\partial V_{\mathrm{b}}}{\partial P_{\mathrm{c}}}\right)_{P_{\mathrm{p}}}, \quad C_{\mathrm{bp}}=\frac{1}{V_{\mathrm{b}}}\left(\frac{\partial V_{\mathrm{b}}}{\partial P_{\mathrm{p}}}\right)_{P_{\mathrm{c}}}, \\
C_{\mathrm{pc}} & =\frac{-1}{V_{\mathrm{p}}}\left(\frac{\partial V_{\mathrm{p}}}{\partial P_{\mathrm{c}}}\right)_{P_{\mathrm{p}}}, \quad C_{\mathrm{pp}}=\frac{1}{V_{\mathrm{p}}}\left(\frac{\partial V_{\mathrm{p}}}{\partial P_{\mathrm{p}}}\right)_{P_{\mathrm{c}}},
\end{aligned}
$$

where $V_{\mathrm{b}}$ is the macroscopic bulk volume, $V_{\mathrm{p}}$ is the pore volume, $P_{\mathrm{p}}$ is the pore pressure, and $P_{\mathrm{c}}$ is the confining pressure, which is also equal to the hydrostatic part of the stress tensor, where the convention is used here that compressive stresses and compressive strains are reckoned as positive numbers. Note also that the confining stress is defined here to include all three of the normal stress components, not only the two lateral stresses, as is done in other contexts. The bulk and pore volumes are related by $V_{\mathrm{b}}-V_{\mathrm{p}}=V_{\mathrm{m}}$, where $V_{\mathrm{m}}$ is the volume of the rock matrix, i.e. the mineral phase. The porosity is given by $\phi=V_{\mathrm{p}} / V_{\mathrm{b}}$.

The bulk and pore strain increments can be expressed in terms of the porous rock compressibilities, as follows: 


$$
\begin{aligned}
\mathrm{d} \varepsilon_{\mathrm{b}} & =\frac{-\mathrm{d} V_{\mathrm{b}}}{V_{\mathrm{b}}}=C_{\mathrm{bc}} \mathrm{d} P_{\mathrm{c}}-C_{\mathrm{bp}} \mathrm{d} P_{\mathrm{p}}, \\
\mathrm{d} \varepsilon_{\mathrm{p}} & =\frac{-\mathrm{d} V_{\mathrm{p}}}{V_{\mathrm{p}}}=C_{\mathrm{pc}} \mathrm{d} P_{\mathrm{c}}-C_{\mathrm{pp}} \mathrm{d} P_{\mathrm{p}} .
\end{aligned}
$$

Use of simple superposition and reciprocity arguments shows that the four coefficients in Eqs. (3) and (4) are related by the following three relationships (Geertsma 1957; Zimmerman et al. 1986):

$$
\begin{gathered}
C_{\mathrm{bc}}-C_{\mathrm{bp}}=C_{\mathrm{m}}, \\
C_{\mathrm{pc}}-C_{\mathrm{pp}}=C_{\mathrm{m}}, \\
C_{\mathrm{bp}}=\phi C_{\mathrm{pc}},
\end{gathered}
$$

where $C_{\mathrm{m}}$ is the compressibility of the solid material (i.e. mineral phase).

The development of a linearised theory of poroelasticity that is valid under non-hydrostatic loadings starts with Hooke's law for an isotropic non-porous material (Jaeger et al. 2007):

$$
\begin{aligned}
\varepsilon_{x x} & =\frac{1}{2 G}\left[\sigma_{x x}-\frac{v}{(1+v)}\left(\sigma_{x x}+\sigma_{y y}+\sigma_{z z}\right)\right], \\
\varepsilon_{y y} & =\frac{1}{2 G}\left[\sigma_{y y}-\frac{v}{(1+v)}\left(\sigma_{x x}+\sigma_{y y}+\sigma_{z z}\right)\right], \\
\varepsilon_{z z} & =\frac{1}{2 G}\left[\sigma_{z z}-\frac{v}{(1+v)}\left(\sigma_{x x}+\sigma_{y y}+\sigma_{z z}\right)\right], \\
\varepsilon_{x y} & =\sigma_{x y} / 2 G, \quad \varepsilon_{x z}=\sigma_{x z} / 2 G, \quad \varepsilon_{y z}=\sigma_{y z} / 2 G,
\end{aligned}
$$

where $\left\{\varepsilon_{x x}, \varepsilon_{y y}, \varepsilon_{z z}\right\}$ are the normal strains, $\left\{\sigma_{x x}, \sigma_{y y}, \sigma_{z z}\right\}$ are the normal stresses, $\left\{\varepsilon_{x y}, \varepsilon_{x z}, \varepsilon_{y z}\right\}$ are the shear strains, $\left\{\sigma_{x y}, \sigma_{x z}, \sigma_{y z}\right\}$ are the shear stresses, $G$ is the shear modulus, and $v$ is Poisson's ratio. Note that these stresses and strains represent incremental changes relative to the initial in situ values. These relations can be written in matrix form as

$$
\varepsilon=\frac{1}{2 G} \boldsymbol{\sigma}-\frac{v}{2 G(1+v)} \operatorname{trace}(\boldsymbol{\sigma}) \mathbf{I} .
$$

where $\mathbf{I}$ is the $3 \times 3$ identity matrix, and $\operatorname{trace}(\boldsymbol{\sigma})=\sigma_{x x}+\sigma_{y y}+\sigma_{z z}$.

The simplest way to extend Hooke's law to poroelastic materials is to start with Eqs. (8-11), and note from Eq. (3) that an incremental change in the pore pressure will lead to an incremental bulk strain of $-C_{\mathrm{bp}} \mathrm{d} P_{\mathrm{p}}$, which in the context of a linearised theory is equivalent to $-C_{\mathrm{bp}} P_{\mathrm{p}}$. If the rock is macroscopically isotropic, then the three normal strains caused by a pore pressure increment must be equal, and so they must each be equal to $-C_{\mathrm{bp}} P_{\mathrm{p}} / 3$. Furthermore, within the context of a linearised theory, an increment in pore pressure cannot cause a shear strain in an isotropic rock. Hence, Hooke's law for an isotropic poroelastic rock takes the form:

$$
\boldsymbol{\varepsilon}=\frac{1}{2 G} \boldsymbol{\sigma}-\frac{v}{2 G(1+v)} \operatorname{trace}(\boldsymbol{\sigma}) \mathbf{I}-\frac{C_{\mathrm{bp}}}{3} P_{\mathrm{p}} \mathbf{I},
$$

where $P_{\mathrm{p}}$ represents the incremental change in pore pressure relative to the initial reservoir pressure, prior to any fluid extraction. In Eq. (13), and subsequent equations, the stresses and strains at each point must be interpreted as average values taken over an "infinitesimal" region that is nevertheless large enough to encompass some suitably large number of grains and pores (Bear 1988). 
Recalling from Eq. (5) that $C_{\mathrm{bp}}=C_{\mathrm{bc}}-C_{\mathrm{m}}$, and noting that $C_{\mathrm{bc}}=1 / K_{\mathrm{bc}}=1 / K$, where $K$ is the macroscopic bulk modulus, Eq. (13) can be written as:

$$
\boldsymbol{\varepsilon}=\frac{1}{2 G} \boldsymbol{\sigma}-\frac{v}{2 G(1+v)} \operatorname{trace}(\boldsymbol{\sigma}) \mathbf{I}-\frac{\alpha}{3 K} P_{\mathrm{p}} \mathbf{I},
$$

where $\alpha$ is the Biot coefficient, defined by (Biot and Willis 1957; Detournay and Cheng 1993)

$$
\alpha=1-\frac{C_{\mathrm{m}}}{C_{\mathrm{bc}}}=1-\frac{K_{\mathrm{bc}}}{K_{\mathrm{m}}}=1-\frac{K}{K_{\mathrm{m}}} .
$$

Taking the trace of both sides of Eq. (14), and recalling the relation $K=2 G(1+v) / 3(1-2 v)$, yields

$$
\operatorname{trace}(\varepsilon)=\frac{1}{3 K} \operatorname{trace}(\sigma)-\frac{\alpha}{K} P_{\mathrm{p}}
$$

The bulk strain is equal to the trace of the strain tensor, and so Eq. (16) shows that

$$
\varepsilon_{\mathrm{b}}=\frac{1}{K}\left[\frac{\operatorname{trace}(\boldsymbol{\sigma})}{3}-\alpha P_{\mathrm{p}}\right]=C_{\mathrm{bc}}\left[\frac{\operatorname{trace}(\boldsymbol{\sigma})}{3}-\alpha P_{\mathrm{p}}\right]
$$

Note that integration of Eq. (3), for the linearised case in which the compressibility coefficients do not vary with stress, followed by use of Eq. (15), yields $\varepsilon_{\mathrm{b}}=C_{\mathrm{bc}}\left(P_{\mathrm{c}}-\alpha P_{\mathrm{p}}\right)$. Hence, under non-hydrostatic stress states, the term $(1 / 3) \operatorname{trace}(\sigma)=\left(\sigma_{x x}+\sigma_{y y}+\sigma_{z z}\right) / 3$ plays the role of the confining pressure, $P_{\mathrm{c}}$.

The equations for the stresses in terms of the strains are found by inverting Eqs. (8-11), which yields

$$
\boldsymbol{\sigma}-\alpha P_{\mathrm{p}} \mathbf{I}=2 G\left[\boldsymbol{\varepsilon}+\frac{v}{1-2 v} \operatorname{trace}(\boldsymbol{\varepsilon}) \mathbf{I}\right]=2 G \boldsymbol{\varepsilon}+\frac{2 G v}{1-2 v} \operatorname{trace}(\boldsymbol{\varepsilon}) \mathbf{I},
$$

where $2 G v /(1-2 v)$ is the Lamé parameter, $\lambda$. This relation can be written in component form as:

$$
\begin{aligned}
& \sigma_{x x}-\alpha P_{\mathrm{p}}=2 G \varepsilon_{x x}+\lambda\left(\varepsilon_{x x}+\varepsilon_{y y}+\varepsilon_{z z}\right), \\
& \sigma_{y y}-\alpha P_{\mathrm{p}}=2 G \varepsilon_{y y}+\lambda\left(\varepsilon_{x x}+\varepsilon_{y y}+\varepsilon_{z z}\right), \\
& \sigma_{z z}-\alpha P_{\mathrm{p}}=2 G \varepsilon_{z z}+\lambda\left(\varepsilon_{x x}+\varepsilon_{y y}+\varepsilon_{z z}\right), \\
& \sigma_{x y}=2 G \varepsilon_{x y}, \quad \sigma_{x z}=2 G \varepsilon_{x z}, \quad \sigma_{y z}=2 G \varepsilon_{y z} .
\end{aligned}
$$

These equations are identical to those for a non-porous isotropic elastic rock, except that the term $\alpha P_{\mathrm{p}}$ is subtracted from each of the normal stresses, to yield the so-called effective stresses. (The effective stress tensor is sometimes denoted by $\sigma^{\prime}=\sigma-\alpha P_{\mathrm{p}} \mathbf{I}$.) Constitutive equations and effective stress coefficients for anisotropic poroelastic rocks have been studied by Carroll (1979), Thompson and Willis (1991), and Cheng (1997). However, the case of anisotropy will not be pursued further in the present paper.

The above equations contain the pore fluid pressure as one of the basic variables. In order to fully incorporate the pore fluid into the theory of poroelasticity and relate it to the changes in pore volume, first consider a region of rock having bulk volume $V_{\mathrm{b}}$, whose pore space contains an amount of fluid having mass $m$. If the density of the pore fluid is $\rho_{f}$, the volume occupied by this fluid is $m / \rho_{\mathrm{f}}$. If the pores are fully saturated, this fluid volume is also equal to the pore volume, $V_{\mathrm{p}}$. The incremental change in the pore volume is therefore given by:

$$
\mathrm{d} V_{\mathrm{p}}=\mathrm{d}\left(m / \rho_{\mathrm{f}}\right)=\frac{\mathrm{d} m}{\rho_{\mathrm{f}}}-\frac{m \mathrm{~d} \rho_{\mathrm{f}}}{\rho_{\mathrm{f}}^{2}}=\frac{\mathrm{d} m}{\rho_{\mathrm{f}}}-\frac{m}{\rho_{\mathrm{f}}} \frac{\mathrm{d} \rho_{\mathrm{f}}}{\rho_{\mathrm{f}}} .
$$


But $\mathrm{d} \rho_{\mathrm{f}} / \rho_{\mathrm{f}}=C_{\mathrm{f}} \mathrm{d} P_{\mathrm{p}}$, where $C_{\mathrm{f}}$ is the compressibility of the pore fluid, and so Eq. (23) can be written as:

$$
\mathrm{d} V_{\mathrm{p}}=\frac{\mathrm{d} m}{\rho_{\mathrm{f}}}-V_{\mathrm{p}} C_{\mathrm{f}} \mathrm{d} P_{\mathrm{p}}
$$

Dividing all terms by the bulk volume gives

$$
\frac{\mathrm{d} V_{\mathrm{p}}}{V_{\mathrm{b}}}=\frac{1}{V_{\mathrm{b}}} \frac{\mathrm{d} m}{\rho_{\mathrm{f}}}-\phi C_{\mathrm{f}} \mathrm{d} P_{\mathrm{p}}
$$

The change in the volumetric fluid content of a given region of rock can therefore be broken up into two parts. The second term on the right represents compression or expansion of the fluid that is already in that region, whereas the first term on the right represents additional fluid moving into the region. This first term is denoted by $\mathrm{d} \zeta$ and is defined as that portion of the change in fluid volume that is due solely to mass transfer, i.e.

$$
\mathrm{d} \zeta \equiv \frac{1}{V_{\mathrm{b}}} \frac{\mathrm{d} m}{\rho}=\frac{\mathrm{d} V_{\mathrm{p}}}{V_{\mathrm{b}}}+\phi C_{\mathrm{f}} \mathrm{d} P_{\mathrm{p}}
$$

Recalling Eq. (4) for the pore volume change, the increment in fluid content, $d \zeta$, can also be expressed as:

$$
\mathrm{d} \zeta=-\phi\left[C_{\mathrm{pc}} \mathrm{d} P_{\mathrm{c}}-\left(C_{\mathrm{pp}}+C_{\mathrm{f}}\right) \mathrm{d} P_{\mathrm{p}}\right]
$$

In an undrained process, the increment in fluid content must by definition be zero. Setting $\mathrm{d} \zeta=0$ in Eq. (27) yields

$$
B=\left(\frac{\mathrm{d} P_{\mathrm{p}}}{\mathrm{d} P_{\mathrm{c}}}\right)_{\zeta}=\frac{C_{\mathrm{pc}}}{C_{\mathrm{pp}}+C_{\mathrm{f}}}=\frac{C_{\mathrm{pp}}+C_{\mathrm{m}}}{C_{\mathrm{pp}}+C_{\mathrm{f}}},
$$

where $B$ is Skempton's induced pore pressure coefficient. Using the relations (5-7) between the porous rock compressibilities, Eq. (27) can also be written as:

$$
\mathrm{d} \zeta=-\alpha C_{\mathrm{bc}}\left(\mathrm{d} P_{\mathrm{c}}-\frac{1}{B} \mathrm{~d} P_{\mathrm{p}}\right)=-\frac{\alpha}{K}\left(\mathrm{~d} P_{\mathrm{c}}-\frac{1}{B} \mathrm{~d} P_{\mathrm{p}}\right) .
$$

Equation (29) can be integrated, making use of the fact, by definition, $\zeta=0$ in the unstressed state, to yield

$$
\zeta=-\frac{\alpha}{K}\left(P_{\mathrm{c}}-\frac{1}{B} P_{\mathrm{p}}\right)
$$

Inverting Eq. (30) by using Eq. (17) to eliminate $P_{\mathrm{c}}$ yields

$$
P_{\mathrm{p}}=\frac{B K}{\alpha(1-\alpha B)}\left(\zeta+\alpha \varepsilon_{\mathrm{b}}\right) \equiv M\left(\zeta+\alpha \varepsilon_{\mathrm{b}}\right),
$$

which expresses the pore pressure in terms of the bulk volumetric strain and the excess fluid content. The parameter $M$, also sometimes denoted by $Q$, is known as the Biot modulus.

\section{Diffusion Equation for the Pore Pressure}

In the previous equations, the pore fluid pressure was treated as an independent variable. This is appropriate for laboratory experiments, in which the pore fluid pressure can be controlled. In field-scale situations, the spatial and temporal evolution of the pore fluid pressure will 
be governed by a partial differential equation. This equation can be derived by considering conservation of mass for the pore fluid and using Darcy's law to relate the fluid flux to the pore pressure gradient.

Consider an imaginary surface in a rock, having area $\mathrm{d} A$, and an outward unit normal vector $\mathbf{n}$. The fluid flux vector, $\mathbf{q}$, with dimensions of $[\mathrm{m} / \mathrm{s}]$, is defined such that the total volume of fluid that passes through this surface, per unit time, is given by $(\mathbf{q} \cdot \mathbf{n}) \mathrm{d} A$, where $\mathbf{q} \cdot \mathbf{n}$ is the component of the flux that is normal to the surface. Now consider a piece of rock that occupies a connected region of space $R$, with outer boundary $\partial R$. The total volumetric flux of fluid leaving this region, per unit time, is given by the integral of $(\mathbf{q} \cdot \mathbf{n}) \mathrm{d} A$ over the entire outer surface. The total increment of fluid volume stored within that region, due to mass transfer across the outer boundary, is found by integrating $\zeta$ over the region. The time rate of change of this integral must equal the total flux into the region, so

$$
\iiint_{R} \frac{\partial \zeta}{\partial t} \mathrm{~d} V+\iint_{\partial R}(\mathbf{q} \cdot \mathbf{n}) \mathrm{d} A=0 .
$$

Using the divergence theorem to transform the surface integral into a volume integral yields

$$
\iiint_{R}\left(\frac{\partial \zeta}{\partial t}+\nabla \cdot \mathbf{q}\right) \mathrm{d} V=0,
$$

where $\nabla \cdot \mathbf{q}=\left(\partial q_{x} / \partial x\right)+\left(\partial q_{y} / \partial y\right)+\left(\partial q_{z} / \partial z\right)$ is the divergence of the fluid flux. Since Eq. (33) must hold for any arbitrary sub-region of $R$, the bracketed integrand must vanish identically at all points of $R$, i.e.

$$
\frac{\partial \zeta}{\partial t}+\nabla \cdot \mathbf{q}=0
$$

For sufficiently low flowrates, the volumetric fluid flux in an hydraulically isotropic rock is proportional to the gradient of the pore pressure gradient, according to Darcy's law:

$$
\mathbf{q}=-\frac{k}{\mu} \nabla P_{\mathrm{p}}
$$

where $k$ is the permeability, and $\mu$ is the fluid viscosity. (The gravity term is ignored here, for simplicity; see Bear (1988) for a discussion of the treatment of that term.) Insertion of Eq. (35) into Eq. (34) yields

$$
\frac{\partial \zeta}{\partial t}=\frac{k}{\mu} \nabla^{2} P_{\mathrm{p}}
$$

If the assumption is made that the rock is rigid and undeformable, then Eq. (27) reduces to $\mathrm{d} \zeta=\phi C_{\mathrm{f}} d P_{\mathrm{p}}$, and Eq. (36) takes the form

$$
\frac{\partial P_{\mathrm{p}}}{\partial t}=\frac{k}{\phi \mu C_{\mathrm{f}}} \nabla^{2} P_{\mathrm{p}} .
$$

Equation (37) is a diffusion equation for the pore pressure, in which the hydraulic diffusivity is given by $D=k / \phi \mu C_{\mathrm{f}}$.

If the rock is deformable, and the confining pressure somehow remains constant, Eq. (27) reduces to $\mathrm{d} \zeta=\phi\left(C_{\mathrm{pp}}+C_{\mathrm{f}}\right) \mathrm{d} P_{\mathrm{p}}$, in which case Eq. (37) takes the form (Marsily 1986)

$$
\frac{\partial P_{\mathrm{p}}}{\partial t}=\frac{k}{\phi \mu\left(C_{\mathrm{f}}+C_{\mathrm{pp}}\right)} \nabla^{2} P_{\mathrm{p}} .
$$


In the general case of a deformable poroelastic rock, making no assumptions about the macroscopic stresses or strains and combining Eq. (27) with Eq. (36), yields

$$
\frac{\partial P_{\mathrm{p}}}{\partial t}=\frac{k M}{\mu} \nabla^{2} P_{\mathrm{p}}+\alpha M \frac{\partial \varepsilon_{\mathrm{b}}}{\partial t} .
$$

This equation for the evolution of the pore pressure can also be expressed entirely in terms of pressures and stresses by using Eq. (17) to eliminate $\varepsilon_{\mathrm{b}}$, to arrive at

$$
\frac{\partial P_{\mathrm{p}}}{\partial t}=\frac{k B K}{\alpha \mu} \nabla^{2} P_{\mathrm{p}}+B \frac{\partial P_{\mathrm{c}}}{\partial t} .
$$

The pore pressure is therefore governed by a diffusion-type equation that contains a source/sink term that couples the change in pore pressure to the change in the confining pressure or the bulk strain. The "source term" appearing in the pressure diffusion equation is due to the induced pore pressure caused by a change in confining stress, caused by the Skempton effect (Cheng 2016).

\section{Pore Volume and Porosity Changes Under Uniaxial Strain Conditions}

When fluid is produced from a reservoir, it is often assumed that the reservoir deforms under conditions of zero lateral strain (Fjaer et al. 2008). Although this is a simplification of the deformation that occurs in a reservoir, the assumption of uniaxial strain is nevertheless highly accurate in many situations (Altmann et al. 2010).

For a non-porous rock, the uniaxial compressibility can be defined as the coefficient that relates the axial strain to the vertical stress $\sigma_{z z}$, with the other two lateral strains held constant. Using Eqs. (8-10), this coefficient can be shown to be given by:

$$
C_{\mathrm{uni}}=\left(\frac{\partial \varepsilon_{z z}}{\partial \sigma_{z z}}\right)_{\varepsilon_{x x}, \varepsilon_{y y}}=\frac{1}{\lambda+2 G}=\frac{(1+v)}{3(1-v) K}=\frac{(1+v)}{3(1-v)} C_{\mathrm{bc}} .
$$

If uniaxial compaction of a porous rock occurs under drained conditions, setting the change in pore pressure equal to zero in Eq. (21) shows that Eq. (41) will continue to hold.

This uniaxial compressibility coefficient may be relevant to compaction that occurs over geological time spans, due to continued deposition of sediments, for example. Of more direct engineering relevance is the deformation caused by withdrawal of fluid from a reservoir, while the lateral strains and the vertical stress are held constant. This coefficient is found directly from Eq. (21) to be (Geertsma 1966):

$$
C_{\text {uni }, p}=-\left(\frac{\partial \varepsilon_{z z}}{\partial P_{\mathrm{p}}}\right)_{\varepsilon_{x x}, \varepsilon_{y y}, \sigma_{z z}}=\frac{\alpha}{\lambda+2 G}=\frac{(1+v)}{3(1-v)} \alpha C_{\mathrm{bc}} .
$$

Comparison of Eqs. (41) and (42) shows that the uniaxial compressibility with respect to changes in pore pressure is equal to the uniaxial compressibility with respect to changes in axial stress, multiplied by the Biot coefficient.

A uniaxial pore volume compressibility can be defined as (Raaen 1993):

$$
C_{\mathrm{pp}}^{\mathrm{uni}}=\frac{1}{V_{\mathrm{p}}}\left(\frac{\partial V_{\mathrm{p}}}{\partial P_{\mathrm{p}}}\right)_{\varepsilon_{x x}, \varepsilon_{y y}, \sigma_{z z}} .
$$


This coefficient can be expressed in terms of the hydrostatic compressibility parameters, by starting with the following identity,

$$
V_{\mathrm{b}}=V_{\mathrm{p}}+V_{\mathrm{m}}
$$

Taking derivatives with respect to pore pressure under conditions of constant lateral strain and constant vertical stress, and recalling the "compression is positive" sign convention, leads to

$$
\left(\frac{\partial \varepsilon_{\mathrm{b}}}{\partial P_{\mathrm{p}}}\right)_{\text {uni }}=\phi\left(\frac{\partial \varepsilon_{\mathrm{p}}}{\partial P_{\mathrm{p}}}\right)_{\text {uni }}+(1-\phi)\left(\frac{\partial \varepsilon_{\mathrm{m}}}{\partial P_{\mathrm{p}}}\right)_{\text {uni }} .
$$

But $\varepsilon_{\mathrm{b}}=\varepsilon_{x x}+\varepsilon_{y y}+\varepsilon_{z z}$, and the two lateral strains are zero, so for uniaxial deformation, $\varepsilon_{\mathrm{b}}=\varepsilon_{z z}$. The first term in parentheses on the right in Eq. (45) is the negative of the uniaxial pore compressibility defined by Eq. (43), so Eq. (45) can be rewritten as:

$$
\phi C_{\mathrm{pp}}^{\mathrm{uni}}=-\left(\frac{\partial \varepsilon_{z z}}{\partial P_{\mathrm{p}}}\right)_{\mathrm{uni}}+(1-\phi)\left(\frac{\partial \varepsilon_{\mathrm{m}}}{\partial P_{\mathrm{p}}}\right)_{\mathrm{uni}} .
$$

The first term on the right of Eq. (46) is the uniaxial compressibility with respect to pore pressure, which is given by Eq. (42). Hence,

$$
C_{\mathrm{pp}}^{\mathrm{uni}}=\frac{(1+v) \alpha C_{\mathrm{bc}}}{3(1-v) \phi}+\frac{(1-\phi)}{\phi}\left(\frac{\partial \varepsilon_{\mathrm{m}}}{\partial P_{\mathrm{p}}}\right)_{\mathrm{uni}} .
$$

The derivative of the matrix strain with respect to pore pressure can be found by starting with the following general equation for the solid mineral phase strain, which holds under hydrostatic or non-hydrostatic stresses (Zimmerman et al. 1994):

$$
\varepsilon_{\mathrm{m}}=C_{\mathrm{m}}\left(\frac{P_{\mathrm{c}}-\phi P_{\mathrm{p}}}{1-\phi}\right) .
$$

Although no vertical stress is applied during this process, the change in pore pressure will induce lateral stresses, due to the lateral constraint and the Poisson effect. These stresses can be found from Eqs. (19-21) to be (Hettema et al. 1998)

$$
\sigma_{x x}=\sigma_{y y}=\frac{(1-2 v) \alpha P_{\mathrm{p}}}{(1-v)} .
$$

But $P_{\mathrm{c}}=\left(\sigma_{x x}+\sigma_{y y}+\sigma_{z z}\right) / 3$, so inserting Eq. (49) into Eq. (48) yields

$$
\varepsilon_{\mathrm{m}}=\frac{\phi}{1-\phi}\left[\frac{2(1-2 v) \alpha}{3(1-v) \phi}-1\right] C_{\mathrm{m}} P_{\mathrm{p}} .
$$

The derivative in Eq. (47) can now be calculated, from Eq. (50), to yield

$$
C_{\mathrm{pp}}^{\mathrm{uni}}=\frac{(1+v) \alpha C_{\mathrm{bc}}}{3(1-v) \phi}+\left[\frac{2(1-2 v) \alpha}{3(1-v) \phi}-1\right] C_{\mathrm{m}} .
$$

Use of Eqs. (5-7) allows the uniaxial pore compressibility to also be expressed as:

$$
C_{\mathrm{pp}}^{\mathrm{uni}}=C_{\mathrm{pp}}-\frac{2(1-2 v) \alpha}{3(1-v)}\left(C_{\mathrm{pp}}+C_{\mathrm{m}}\right) .
$$

This latter expression shows that lateral confinement causes the pore compressibility to be lower than it would be under hydrostatic conditions. As it is typically the case that $C_{\mathrm{pp}} \gg C_{\mathrm{m}}$ 
(Laurent et al. 1993), the ratio of uniaxial to hydrostatic pore compressibility is approximately given by

$$
\frac{C_{\mathrm{pp}}^{\mathrm{uni}}}{C_{\mathrm{pp}}^{\mathrm{hydro}}} \approx 1-\frac{2(1-2 v) \alpha}{3(1-v)} .
$$

For typical values for a consolidated sandstone (Detournay and Cheng 1993), such as $\alpha=0.7$ and $v=0.15$, Eq. (53) shows that the uniaxial pore volume compressibility will be about three-fifths as large as the hydrostatic pore volume compressibility.

As pointed out by Raaen (1993), it is often assumed, by an unwarranted analogy with Eq. (42) for the bulk volume compressibility, that the ratio of uniaxial pore volume compressibility to hydrostatic pore volume compressibility will be $(1+v) / 3(1-v)$. Comparison with the exact expression (52) shows that this assumption may lead to errors of up to $40 \%$, when converting hydrostatic pore volume compressibilities to uniaxial values (see data in Zimmerman 2000b).

A uniaxial version of $C_{\mathrm{pc}}$ can be defined, with respect to changes in vertical overburden stress, as

$$
C_{\mathrm{pc}}^{\mathrm{uni}}=\left(\frac{\partial \varepsilon_{\mathrm{p}}}{\partial \sigma_{z z}}\right)_{\varepsilon_{x x}, \varepsilon_{y y}, P_{\mathrm{p}}} .
$$

But $\varepsilon_{\mathrm{p}}=C_{\mathrm{pc}} P_{\mathrm{c}}-C_{\mathrm{pp}} P_{\mathrm{p}}$, so

$$
C_{\mathrm{pc}}^{\mathrm{uni}}=C_{\mathrm{pc}}\left(\frac{\partial P_{\mathrm{c}}}{\partial \sigma_{z z}}\right)_{\varepsilon_{x x}, \varepsilon_{y y}, P_{\mathrm{p}}}-C_{\mathrm{pp}}\left(\frac{\partial P_{\mathrm{p}}}{\partial \sigma_{z z}}\right)_{\varepsilon_{x x}, \varepsilon_{y y}, P_{\mathrm{p}}} .
$$

The second derivative on the right is zero, by definition, since $P_{\mathrm{p}}$ is held constant in this process. Equations (19-21) show that during uniaxial strain, with pore pressure held constant, $\sigma_{x x}=\sigma_{y y}=\lambda \varepsilon_{z z}$, and $\sigma_{z z}=(\lambda+2 G) \varepsilon_{z z}$. Hence, $P_{\mathrm{c}}=\left(\sigma_{x x}+\sigma_{y y}+\sigma_{z z}\right) / 3=(3 \lambda+$ $2 G) \varepsilon_{z z} / 3=(3 \lambda+2 G) \sigma_{z z} / 3(\lambda+2 G)$, and so the first derivative on the right is given by:

$$
\left(\frac{\partial P_{\mathrm{c}}}{\partial \sigma_{z z}}\right)_{\varepsilon_{x x}, \varepsilon_{y y}, P_{\mathrm{p}}}=\frac{3 \lambda+2 G}{3(\lambda+2 G)}=\frac{1+v}{3(1-v)} .
$$

Substituting Eq. (56) into Eq. (55) yields

$$
C_{\mathrm{pc}}^{\mathrm{uni}}=\frac{1+v}{3(1-v)} C_{\mathrm{pc}}^{\mathrm{hydro}}
$$

The various elements are now in place to derive a master equation for the change in porosity that results from changes in the pore pressure and/or the vertical overburden stress, under conditions of uniaxial strain. Starting with $\phi=V_{\mathrm{p}} / V_{\mathrm{b}}$, and taking the logarithmic derivative, yields

$$
\frac{\mathrm{d} \phi}{\phi}=\frac{\mathrm{d} V_{\mathrm{p}}}{V_{\mathrm{p}}}-\frac{\mathrm{d} V_{\mathrm{b}}}{V_{\mathrm{b}}}=-\varepsilon_{\mathrm{p}}+\varepsilon_{\mathrm{b}} .
$$

Each of the two strain terms on the right side will depend on the increment in vertical stress and the increment in pore pressure. The coefficient that relates pore strain to vertical stress is given by Eq. (57), the coefficient that relates pore strain to pore pressure is given by Eq. (51), the coefficient that relates bulk strain to vertical stress is given by Eq. (41), and the coefficient that relates bulk strain to pore pressure is given by Eq. (42). Inserting all four of these differential terms into Eq. (58) yields, after multiplying through by $\phi$ : 


$$
\begin{aligned}
\mathrm{d} \phi= & -\left[\frac{(1+v) \phi C_{\mathrm{pc}}}{3(1-v)}\right] \mathrm{d} \sigma_{z z}+\left\{\frac{(1+v) \alpha C_{\mathrm{bc}}}{3(1-v)}+\left[\frac{2(1-2 v) \alpha}{3(1-v)}-\phi\right] C_{\mathrm{m}}\right\} \mathrm{d} P_{\mathrm{p}} \\
& +\left[\frac{(1+v) \phi C_{\mathrm{bc}}}{3(1-v)}\right] \mathrm{d} \sigma_{z z}-\left[\frac{(1+v) \alpha \phi C_{\mathrm{bc}}}{3(1-v)}\right] \mathrm{d} P_{\mathrm{p}} \\
= & {\left[\frac{(1+v) \phi\left(C_{\mathrm{bc}}-C_{\mathrm{pc}}\right)}{3(1-v)}\right] \mathrm{d} \sigma_{z z}+\left\{\frac{(1+v) \alpha C_{\mathrm{bc}}(1-\phi)}{3(1-v)}+\left[\frac{2(1-2 v) \alpha}{3(1-v)}-\phi\right] C_{\mathrm{m}}\right\} \mathrm{d} P_{\mathrm{p}} . }
\end{aligned}
$$

Using relations $(5-7,15)$, this equation for the porosity change can also be written as

$$
\begin{aligned}
\mathrm{d} \phi= & -\left\{\frac{(1+v)\left[C_{\mathrm{bc}}(1-\phi)-C_{\mathrm{m}}\right]}{3(1-v)}\right\} \mathrm{d} \sigma_{z z}+\left\{\frac{(1+v) \alpha C_{\mathrm{bc}}(1-\phi)}{3(1-v)}\right. \\
& \left.+\left[\frac{2(1-2 v) \alpha}{3(1-v)}-\phi\right] C_{\mathrm{m}}\right\} \mathrm{d} P_{\mathrm{p}}
\end{aligned}
$$

It is worthwhile to compare this new equation, derived within the context of linearised poroelasticity, with the widely used equation for the porosity change that was suggested by Palmer and Mansoori (1998). In the present notation, making use of Eq. (15) where convenient, their equation can be written as:

$\mathrm{d} \phi=-\left[\frac{(1+v) C_{\mathrm{bc}}}{3(1-v)}-(1-\phi) f C_{\mathrm{m}}\right] \mathrm{d} \sigma_{z z}+\left[\frac{(1+v) \alpha C_{\mathrm{bc}}}{3(1-v)}+(1-\phi)(1-f) C_{\mathrm{m}}\right] \mathrm{d} P_{\mathrm{p}}$.

where $f$ is a parameter that can vary between 0 and 1 , and which, according to Moore et al. (2015), is related to the ratio of isotropic strain to deviatoric strain within the mineral grains. Although there is no choice of the $f$ parameter that can cause expressions (60) and (61) to become identical, it is interesting to note that, in the limit when the porosity of the rock is low, and the Biot coefficient is close to 1 (which corresponds to a very compressible porous rock), the two expressions will coincide if $f$ is taken to be

$$
f=\frac{1+v}{3(1-v)} \text {. }
$$

\section{Effect of Temperature on Porosity Under Uniaxial Strain Conditions}

It is also of interest to have access to expressions for the changes in axial strain, pore volume, and porosity, caused by, say, a uniform change in temperature in a reservoir. Before deriving these expressions, it worth recalling that, if a micro-homogeneous porous material is heated, in the absence of any external constraints, the pore volume and bulk volume will each expand by the same fractional amount, and the porosity will remain unchanged (Cheng 2016, Eq. 11.43). Moreover, the drained thermal expansion coefficient of the porous material, which is the primitive coefficient that naturally appears in the constitutive equations, will be exactly equal to the thermal expansion coefficient of the non-porous mineral phase (McTigue 1986; Cheng 2016, Eq. 11.54). But if a porous material is heated while subject to the constraint of uniaxial strain, lateral stresses will be induced, due to the Poisson effect, and these stresses will in turn lead to a change in porosity. The coefficient that relates this change in porosity to the change in temperature can be found from an analysis similar to that carried out in Sect. 4, but now using the thermoporoelastic version of Hooke's law. 
Hooke's law for an isotropic, porous, thermoelastic rock is found from the poroelastic version by simply subtracting a term $\beta \theta$ from each normal strain (Zimmerman 2000a):

$$
\begin{aligned}
\varepsilon_{x x} & =\frac{1}{2 G}\left[\sigma_{x x}-\frac{v}{(1+v)}\left(\sigma_{x x}+\sigma_{y y}+\sigma_{z z}\right)\right]-\frac{\alpha P_{\mathrm{p}}}{3 K}-\beta \theta, \\
\varepsilon_{y y} & =\frac{1}{2 G}\left[\sigma_{y y}-\frac{v}{(1+v)}\left(\sigma_{x x}+\sigma_{y y}+\sigma_{z z}\right)\right]-\frac{\alpha P_{\mathrm{p}}}{3 K}-\beta \theta, \\
\varepsilon_{z z} & =\frac{1}{2 G}\left[\sigma_{z z}-\frac{v}{(1+v)}\left(\sigma_{x x}+\sigma_{y y}+\sigma_{z z}\right)\right]-\frac{\alpha P_{\mathrm{p}}}{3 K}-\beta \theta, \\
\varepsilon_{x y} & =\sigma_{x y} / 2 G, \quad \varepsilon_{x z}=\sigma_{x z} / 2 G, \quad \varepsilon_{y z}=\sigma_{y z} / 2 G .
\end{aligned}
$$

where $\beta$ is the linear thermal expansion coefficient, and $\theta=T-T_{o}$ is the temperature increment above some initial baseline temperature $T_{o}$, at which the strains are considered to be zero. The minus signs again appear due to the fact that extensile strains are considered to be negative. Although the thermal expansion coefficient is usually denoted by $\alpha$, in the present paper it will be denoted by $\beta$, since $\alpha$ is used here for the Biot coefficient.

To find the coupling coefficients, which relate changes in temperature to changes in the strains, porosity, etc., the pore pressure terms in Eqs. (63-65) can be ignored, as pore pressure will be held constant in the processes now being considered. This can be viewed as corresponding either to a fully drained process, or an undrained situation in which the pore fluid is sufficiently compressible that a temperature change induces a negligible increase in pore pressure (Palmer and Mansoori 1998). Furthermore, Eq. (66) can be ignored, as the volumetric strains do not involve the shear strain components. Equations (63-65) can be inverted to give the stresses as functions of the strains:

$$
\begin{aligned}
\sigma_{x x} & =2 G \varepsilon_{x x}+\frac{2 G v}{1-2 v}\left(\varepsilon_{x x}+\varepsilon_{y y}+\varepsilon_{z z}\right)+3 \beta K \theta, \\
\sigma_{y y} & =2 G \varepsilon_{y y}+\frac{2 G v}{1-2 v}\left(\varepsilon_{x x}+\varepsilon_{y y}+\varepsilon_{z z}\right)+3 \beta K \theta, \\
\sigma_{z z} & =2 G \varepsilon_{z z}+\frac{2 G v}{1-2 v}\left(\varepsilon_{x x}+\varepsilon_{y y}+\varepsilon_{z z}\right)+3 \beta K \theta .
\end{aligned}
$$

Consider now a state of uniaxial strain in the vertical $z$-direction, i.e. $\varepsilon_{x x}=\varepsilon_{y y}=0$, $\sigma_{z z}=0$, and $P_{\mathrm{p}}=0$, in which case the resultant stresses and strains are due only to the temperature increment. In this situation, Eq. (69) shows that vertical strain, which is the only nonzero strain, is given by

$$
\varepsilon_{z z}=-\frac{3 \beta K \theta(1-2 v)}{2 G(1-v)}=-\frac{(1+v)}{(1-v)} \beta \theta .
$$

Since the two lateral strains are zero, the bulk volumetric strain is equal to the vertical strain:

$$
\varepsilon_{\mathrm{b}}=-\frac{(1+v)}{(1-v)} \beta \theta .
$$

The change in porosity can be found by appropriately combining the bulk volumetric strain and the strain in the mineral grains. First, note that, by definition,

$$
\phi=\frac{V_{\mathrm{p}}}{V_{\mathrm{b}}}=\frac{V_{\mathrm{b}}-V_{\mathrm{m}}}{V_{\mathrm{b}}}=1-\frac{V_{\mathrm{m}}}{V_{\mathrm{b}}} .
$$


Taking differentials yields

$$
\mathrm{d} \phi=-\frac{\mathrm{d} V_{\mathrm{m}}}{V_{\mathrm{b}}}+\frac{V_{\mathrm{m}}}{V_{\mathrm{b}}^{2}} \mathrm{~d} V_{\mathrm{b}}=-\frac{V_{\mathrm{m}}}{V_{\mathrm{b}}} \frac{\mathrm{d} V_{\mathrm{m}}}{V_{\mathrm{m}}}+\frac{V_{\mathrm{m}}}{V_{\mathrm{b}}} \frac{\mathrm{d} V_{\mathrm{b}}}{V_{\mathrm{b}}}=-(1-\phi)\left(\varepsilon_{\mathrm{b}}-\varepsilon_{\mathrm{m}}\right) .
$$

The bulk strain is given by Eq. (71), and so an expression for the strain in the mineral phase is needed in order to find the change in porosity.

Consider now the thermoelastic Hooke's law applied at the micro-scale, for the mineral phase:

$$
\begin{aligned}
& \varepsilon_{x x}=\frac{1}{2 G_{m}}\left[\tau_{x x}-\frac{v_{m}}{\left(1+v_{m}\right)}\left(\tau_{x x}+\tau_{y y}+\tau_{z z}\right)\right]-\beta \theta, \\
& \varepsilon_{y y}=\frac{1}{2 G_{m}}\left[\tau_{y y}-\frac{v_{m}}{\left(1+v_{m}\right)}\left(\tau_{x x}+\tau_{y y}+\tau_{z z}\right)\right]-\beta \theta, \\
& \varepsilon_{z z}=\frac{1}{2 G_{m}}\left[\tau_{z z}-\frac{v_{m}}{\left(1+v_{m}\right)}\left(\tau_{x x}+\tau_{y y}+\tau_{z z}\right)\right]-\beta \theta,
\end{aligned}
$$

where the subscript $m$ is used for the elastic moduli of the mineral phase, but none is needed for the thermal expansion coefficient of the mineral phase, $\beta_{\mathrm{m}}$, as it is identical to the thermal expansion coefficient of the porous rock, $\beta$. Note that these stresses and strains are defined at the micro-scale, so that, in a sandstone, for example, they would vary within each sand grain. This is in contrast the "macroscopic" stresses and strains used in previous sections, which were averaged out over a suitable REV.

The local volumetric strain in the mineral phase can be found by summing Eqs. (74-76):

$$
\begin{aligned}
\varepsilon_{\mathrm{m}} & =\varepsilon_{x x}+\varepsilon_{y y}+\varepsilon_{z z}=\frac{1}{2 G_{m}} \frac{\left(1-2 v_{m}\right)}{\left(1+v_{m}\right)}\left(\sigma_{x x}+\sigma_{y y}+\sigma_{z z}\right)-3 \beta \theta \\
& =\frac{1}{K_{\mathrm{m}}} \frac{\left(\sigma_{x x}+\sigma_{y y}+\sigma_{z z}\right)}{3}-3 \beta \theta \\
& =\frac{1}{K_{\mathrm{m}}} \frac{\operatorname{tr}(\boldsymbol{\sigma})}{3}-3 \beta \theta .
\end{aligned}
$$

Averaging the local volumetric bulk strain over the entire mineral phase yields

$$
\left\langle\varepsilon_{\mathrm{m}}\right\rangle=\frac{1}{K_{\mathrm{m}}} \frac{\langle\operatorname{tr}(\boldsymbol{\sigma})\rangle}{3}-3 \beta\langle\theta\rangle .
$$

The temperature increment is assumed to be uniform throughout the porous body, so $\langle\theta\rangle=\theta$. The term $\langle\operatorname{tr}(\sigma)\rangle / 3$ is the spatial average of the mean normal stress, averaged throughout the mineral phase. This quantity is related to the externally applied confining pressure, $P_{\mathrm{c}}$, and the pore pressure, $P_{\mathrm{p}}$, by (Zimmerman et al. 1994, Eq. A8)

$$
\frac{\langle\operatorname{tr}(\sigma)\rangle}{3}=\frac{P_{\mathrm{c}}-\phi P_{\mathrm{p}}}{1-\phi} .
$$

As no pore pressure increment is being considered in this process, combining Eqs. (78) and (79) yields

$$
\left\langle\varepsilon_{\mathrm{m}}\right\rangle=\frac{P_{\mathrm{c}}}{K_{\mathrm{m}}(1-\phi)}-3 \beta \theta .
$$

The term $P_{\mathrm{c}}$ is, by definition, the mean value of the three macroscopic normal stresses, i.e. $P_{\mathrm{c}}=\left(\sigma_{x x}+\sigma_{y y}+\sigma_{z z}\right) / 3$. Under uniaxial strain conditions, noting that the stresses in this discussion are all incremental stresses, $\sigma_{z z}=0$, and Eqs. (67-68) show that 


$$
\sigma_{x x}=\sigma_{y y}=\frac{2 G v}{1-2 v} \varepsilon_{z z}+3 \beta K \theta,
$$

from which it follows that

$$
P_{\mathrm{c}}=\frac{\sigma_{x x}+\sigma_{y y}+\sigma_{z z}}{3}=\frac{4 G v}{3(1-2 v)} \varepsilon_{z z}+2 \beta K \theta .
$$

Recalling Eq. (70) for the vertical strain, Eq. (82) can be written as

$$
P_{\mathrm{c}}=-\frac{4 G v}{3(1-2 v)} \frac{(1+v)}{(1-v)} \beta \theta+2 \beta K \theta=-\frac{2 K v}{(1-v)} \beta \theta+2 \beta K \theta .
$$

Inserting this expression into Eq. (80) yields

$$
\left\langle\varepsilon_{\mathrm{m}}\right\rangle=-\frac{2 K v}{(1-v)} \frac{\beta \theta}{K_{\mathrm{m}}(1-\phi)}+\frac{2 \beta K \theta}{K_{\mathrm{m}}(1-\phi)}-3 \beta \theta .
$$

But $K / K_{\mathrm{m}}=1-\alpha$, where $\alpha$ is the Biot coefficient, so:

$$
\begin{aligned}
\left\langle\varepsilon_{\mathrm{m}}\right\rangle & =-\frac{2 v(1-\alpha)}{(1-v)(1-\phi)} \beta \theta+\frac{2(1-\alpha)}{(1-\phi)} \beta \theta-3 \beta \theta \\
& =\frac{2(1-\alpha)}{(1-\phi)} \beta \theta\left[1-\frac{v}{1-v}\right]-3 \beta \theta \\
& =\frac{2(1-\alpha)}{(1-\phi)} \frac{(1-2 v)}{(1-v)} \beta \theta-3 \beta \theta .
\end{aligned}
$$

Finally, inserting the bulk strain from Eq. (71), and the mineral strain from Eq. (85), into Eq. (73), yields

$$
\begin{aligned}
\mathrm{d} \phi & =(1-\phi)\left[\frac{(1+v)}{(1-v)} \beta \theta+\frac{2(1-\alpha)}{(1-\phi)} \frac{(1-2 v)}{(1-v)} \beta \theta-3 \beta \theta\right] \\
& =(1-\phi)\left[\frac{(1+v)}{3(1-v)}+\frac{2(1-\alpha)}{(1-\phi)} \frac{(1-2 v)}{3(1-v)}-1\right] 3 \beta \theta \\
& =-\left[\frac{2(1-2 v)(\alpha-\phi)}{3(1-v)}\right] \beta \theta .
\end{aligned}
$$

Since the Biot coefficient $\alpha$ must always be greater than the porosity $\phi$ (Zimmerman et al. 1986), this expression shows that an increase in temperature causes a decrease in porosity, as discussed by Palmer and Mansoori (1998).

This new expression, derived rigorously within the context of linearised thermoporoelasticity, can be compared with the expression that was presented without derivation by Palmer and Mansoori (1998), which can be written in the present notation as

$$
\mathrm{d} \phi=-\left[\frac{3(1-v)(1-\phi)-(1+v)}{3(1-v)}\right] \beta \theta .
$$

Although the two expressions differ, Palmer and Mansoori's heuristic expression, Eq. (87), numerically approaches the exact relation, Eq. (86), in the limit of low porosity and high Biot coefficient and coincides with it when $\phi \rightarrow 0$ and $\alpha \rightarrow 1$. 


\section{Diffusion Equation for the Pore Pressure Under Uniaxial Strain Conditions}

As shown in Sect. 3, if the rock formation is rigid and non-deformable, the pore pressure will obey a diffusion equation, with a hydraulic diffusivity given by $D=k / \phi \mu C_{\mathrm{f}}$, where the product $\phi C_{\mathrm{f}}$ is referred to as the storativity. The approximation of a rigid reservoir may be acceptable in some situations. But it has long been recognised in the oil and gas industry that, for most reservoirs, the "formation compressibility" also contributes to the storativity.

Most early discussions of this issue were imprecise with regard to the appropriate "formation compressibility" term that would enter into the storativity. Matthews and Russell (1967, p. 7), in a derivation that implicitly assumed that the pore volume could change while the bulk volume remains constant, arrived at a formation compressibility of $(1 / \phi)\left(\mathrm{d} \phi / \mathrm{d} P_{\mathrm{p}}\right)$. (Note that in much of the petroleum engineering literature, the "formation compressibility" is denoted by $C_{\mathrm{f}}$, whereas in the present paper, the symbol $C_{\mathrm{f}}$ always refers to the fluid compressibility.) However, this assumption of a compliant pore space in a macroscopically rigid reservoir is physically inconsistent.

Chierici (1994, p. 60, p. 141) derived a "formation compressibility" that is given by, in the present notation $(1-\phi) C_{\mathrm{pp}}-\phi C_{\mathrm{m}}$; this term was then added to the fluid compressibility to yield a "total compressibility". Marsily (1986), in a rigorous and elegant treatment aimed at hydrogeologists, considered the case in which the confining stress does not vary during fluid extraction. In the present notation, this assumption leads to Eq. (38) of Sect. 3, in which the hydrostatic pore volume compressibility is added to the fluid compressibility in the diffusivity term. But this type of boundary condition does not correspond to the uniaxial strain conditions that typically occur during depletion of oil and gas reservoirs. Although in more recent years it has often been asserted that the uniaxial pore volume compressibility, rather than the hydrostatic pore volume compressibility, should be added to the fluid compressibility in the diffusion equation, no derivation seems yet to have been given to show that this assumption is consistent with the equations that result from a rigorous poroelastic analysis. Such a derivation will now be given, based on the equations presented in the previous sections of this paper.

From Eq. (49) and the definition $P_{\mathrm{c}}=\left(\sigma_{x x}+\sigma_{y y}+\sigma_{z z}\right) / 3$, it follows that, under uniaxial strain, $P_{\mathrm{c}}=2(1-2 v) \alpha P_{\mathrm{p}} / 3(1-v)$. Inserting this result into the general Eq. (40), which is valid for all deformations and processes, yields

$$
\left[1-\frac{2(1-2 v) \alpha B}{3(1-v)}\right] \frac{\partial P_{\mathrm{p}}}{\partial t}=\frac{k B K}{\alpha \mu} \nabla^{2} P_{\mathrm{p}}
$$

Using Eq. (28) to express $B$ in terms of the various compressibility coefficients leads to

$$
\left[1-\frac{2(1-2 v) \alpha\left(C_{\mathrm{pp}}+C_{\mathrm{m}}\right)}{3(1-v)\left(C_{\mathrm{pp}}+C_{\mathrm{f}}\right)}\right] \frac{\partial P_{\mathrm{p}}}{\partial t}=\frac{k\left(C_{\mathrm{pp}}+C_{\mathrm{m}}\right) K}{\alpha \mu\left(C_{\mathrm{pp}}+C_{\mathrm{f}}\right)} \nabla^{2} P_{\mathrm{p}} .
$$

Multiplying both sides by the term $\left(C_{\mathrm{pp}}+C_{\mathrm{f}}\right)$ gives

$$
\left[C_{\mathrm{f}}+C_{\mathrm{pp}}-\frac{2(1-2 v) \alpha\left(C_{\mathrm{pp}}+C_{\mathrm{m}}\right)}{3(1-v)}\right] \frac{\partial P_{\mathrm{p}}}{\partial t}=\frac{k\left(C_{\mathrm{pp}}+C_{\mathrm{m}}\right) K}{\alpha \mu} \nabla^{2} P_{\mathrm{p}} .
$$

Now consider the right-hand side of Eq. (90). According to definition (15), $\alpha=\left(C_{\mathrm{bc}}-\right.$ $\left.C_{\mathrm{m}}\right) / C_{\mathrm{bc}}$. But Eq. (8) shows that $C_{\mathrm{bc}}-C_{\mathrm{m}}=C_{\mathrm{bp}}$, and so $\alpha=C_{\mathrm{bp}} / C_{\mathrm{bc}}$, which, combined with Eq. (7), shows that $\alpha=\phi C_{\mathrm{pc}} / C_{\mathrm{bc}}$. Furthermore, $K=1 / C_{\mathrm{bc}}$ by definition, and so $K / \alpha=K C_{\mathrm{bc}} / \phi C_{\mathrm{pc}}=1 / \phi C_{\mathrm{pc}}$. In the numerator, the term $C_{\mathrm{pp}}+C_{\mathrm{m}}$ is equal to $C_{\mathrm{pc}}$, 
according to Eq. (6). Hence, the coefficient in front of the Laplacian term on the right-hand side of Eq. (90) is simply equal to $k / \mu \phi$, and this equation can therefore be written as

$$
\frac{\partial P_{\mathrm{p}}}{\partial t}=\frac{k}{\mu \phi\left[C_{\mathrm{f}}+C_{\mathrm{pp}}-\frac{2(1-2 v) \alpha}{3(1-v)}\left(C_{\mathrm{pp}}+C_{\mathrm{m}}\right)\right]} \nabla^{2} P_{\mathrm{p}} .
$$

Comparison with Eq. (52) shows that the term added to the fluid compressibility in the denominator of Eq. (91) is nothing other than the uniaxial pore volume compressibility, $C_{\mathrm{pp}}^{\mathrm{uni}}$. This completes the first demonstration, within the context of the fully coupled theory of poroelasticity, that under conditions of uniaxial strain, the pore fluid pressure is governed by a pure diffusion equation of the form

$$
\frac{\partial P_{\mathrm{p}}}{\partial t}=\frac{k}{\phi \mu\left(C_{\mathrm{f}}+C_{\mathrm{pp}}^{\text {uni }}\right)} \nabla^{2} P_{\mathrm{p}} .
$$

A similar equation was suggested by Terzaghi in the context of his famous "consolidation problem" of soil mechanics, under the assumption of an incompressible pore fluid; see Terzaghi et al. (1996) and Jaeger et al. (2007), Sect. 7.6.

Although it may seem obvious that "the pore compressibility term that appears in the pressure diffusion equation should be the uniaxial pore volume compressibility (UPVC)", it must be remembered that the UPVC is not a basic parameter in any of the myriad versions of the theory of linearised poroelasticity. On the contrary, manipulations of the coupled equations governing mechanical deformation and fluid flow lead to a very complicated expression for the compressibility term that contributes to the storativity, as given in Eq. (88), in terms of more "basic" parameters such as the Biot and Skempton coefficients, and it is not obvious that this term is equivalent to the UPVC that would be measured in a static compressibility test. The derivation given above, which required several pages of mathematical manipulations, shows that whereas the final result is perhaps not surprising, it is neither trivial nor self-evident.

\section{Summary and Conclusions}

During production of fluids from an oil or gas reservoir, the deformation is generally assumed to occur under conditions of uniaxial vertical strain. Under these conditions, the changes that occur in the pore volume, and the porosity, will each depend on the increments in the pore pressure, $P_{p}$, the overburden stress, $\sigma_{z z}$, and the temperature, $\theta$. The coefficients that couple the changes in $\left\{P_{p}, \sigma_{z z}, \theta\right\}$ to the resultant changes in pore volume and porosity have been derived within the context of the linearised theory of porothermoelasticity, for microhomogeneous porous media, without invoking any additional assumptions or approximations. The changes in pore volume are of great interest for material balance calculations, whereas the changes in porosity are needed as input for models that relate changes in porosity to changes in permeability. These latter models are of particular importance in coalbed methane reservoirs, the permeabilities of which are highly stress sensitive (Seidle et al. 1992; Shi and Durucan 2004; Moore et al. 2015).

Comparison of the new equation for the porosity change as a function of pore pressure, with the commonly used Palmer-Mansoori equation (Palmer and Mansoori 1998), sheds light on their undefined $f$ parameter and shows that in the limit of low porosity and high Biot coefficient, it is equal to $(1+v) / 3(1-v)$. Assuming a Poisson ratio for coal of 0.45 , this expression is consistent with the value of $f=0.9$ that has been recently been suggested by Moore et al. (2015). Comparison of the newly derived equation for the porosity change 
as a function of temperature, with the porosity-temperature equation suggested by Palmer and Mansoori, shows that their equation is again an approximation to the exact result, but becomes increasingly accurate in the limit of low porosity and high Biot coefficient.

It should be reiterated that these above-mentioned results, Eqs. (60) and (86), have been derived under the assumption that the porous rock is elastically isotropic, and homogeneous on the grain scale - the latter being the so-called ideal porous medium assumption (Cheng 2016).

Finally, it has been shown, apparently for the first time, that under conditions of uniaxial strain, the partial differential equation that governs the evolution of the pore pressure is a pure diffusion equation, with a total compressibility term that (exactly) equals the sum of the fluid compressibility and the uniaxial pore volume compressibility.

Acknowledgements The author thanks Simon Mathias of Durham University for useful discussions of this topic.

Open Access This article is distributed under the terms of the Creative Commons Attribution 4.0 International License (http://creativecommons.org/licenses/by/4.0/), which permits unrestricted use, distribution, and reproduction in any medium, provided you give appropriate credit to the original author(s) and the source, provide a link to the Creative Commons license, and indicate if changes were made.

\section{References}

Altmann, J.B., Müller, T.M., Müller, B.I.R., Tingay, M.R.P., Heidbach, O.: Poroelastic contribution to the reservoir stress path. Int. J. Rock Mech. Min. Sci. 47, 1104-1113 (2010)

Bear, J.: Dynamics of Fluids in Porous Media. Dover, New York (1988)

Biot, M.A.: General theory of three-dimensional consolidation. J. Appl. Phys. 12, 155-164 (1941)

Biot, M.A., Willis, D.G.: The elastic coefficients of the theory of consolidation. J. Appl. Mech. 24, 594-601 (1957)

Carroll, M.M.: An effective stress law for anisotropic elastic deformation. J. Geophys. Res. 84, 7510-7512 (1979)

Cheng, A.H.-D.: Material coefficients of anisotropic poroelasticity. Int. J. Rock Mech. Min Sci. 34, 199-205 (1997)

Cheng, A.H.-D.: Poroelasticity. Springer, Berlin (2016)

Chierici, G.L.: Principles of Petroleum Reservoir Engineering, vol. 1. Springer, Berlin (1994)

de Marsily, G.: Quantitative Hydrogeology. Academic Press, San Diego (1986)

Detournay, E., Cheng, A.H.-D.: Fundamentals of poroelasticity. In: Hudson, J.A. (ed.) Comprehensive Rock Engineering, pp. 113-171. Pergamon Press, Oxford (1993)

Fjaer, E., Holt, R.M., Horsrud, P., Raaen, A.M., Risnes, R.: Petroleum Related Rock Mechanics, 2nd edn. Elsevier, Amsterdam (2008)

Frenkel, J.: On the theory of seismic and seismoelectric phenomena in a moist soil. J. Phys. USSR 8, 230-241 (1944)

Geertsma, J.: A remark on the analogy between thermoelasticity and the elasticity of saturated porous media. J. Mech. Phys. Solids 6, 13-16 (1957)

Geertsma, J.: Problems of rock mechanics in petroleum production engineering. In: Proceedings of 1st Congress International Society Rock Mechanics, pp. 585-594. Lisbon, 25 Sept-1 Oct 1966 (1966)

Hettema, M.H. H., Schutjens, P.M.T.M., Verboom, B.J.M., Gussinklo, H.J.: Production-induced compaction of sandstone reservoirs: the strong influence of field stress. In: Proceedings of the European Petroleum Conference, pp. 547-554. Den Haag, 20-22 Oct (1998)

Jaeger, J.C., Cook, N.G.W., Zimmerman, R.W.: Fundamentals of Rock Mechanics, 4th edn. Wiley, Oxford (2007)

Laurent, J., Boutéca, M.J., Sarda, J.-P., Bary, D.: Pore-pressure influence in the poroelastic behavior of rocks: experimental studies and results. SPE Form. Eval. 9, 117-122 (1993)

Matthews, C.S., Russell, D.G.: Pressure Buildup and Flow Tests in Wells. Society of Petroleum Engineers, Dallas (1967)

McTigue, D.F.: Thermoelastic response of fluid-saturated porous rock. J. Geophys. Res. 91, 9533-9542 (1986) 
Moore, R., Palmer, I., Higgs, N.: Anisotropic model for permeability change in coalbed-methane wells. SPE Reserv. Eval. Eng. 18, 456-462 (2015)

Palmer, I., Mansoori, J.: How permeability depends on stress and pore pressure in coalbeds: a new model. SPE Reserv. Eval. Eng. 1, 539-544 (1998)

Raaen, A.M.: Pore volume compressibility under uniaxial strain conditions from Biot-Gassmann theory. In: Proceedings of 15th European Forms Evaluation Symposium, pp. 1-9. Stavanger, 5-7 May 1993 (1993)

Rice, J.R., Cleary, M.P.: Some basic stress diffusion solutions for fluid saturated elastic porous media with compressible constituents. Rev. Geophys. Space Phys. 14, 227-241 (1976)

Seidle, J.P., Jeansonne, M.W., Erickson, D.J.: Application of matchstick geometry to stress dependent permeability in coals. In: Proceedings of the SPE Rocky Mountain Regional Meeting, Paper SPE 24361. Casper, 18-21 May 1992 (1992)

Shi, J.Q., Durucan, S.: Drawdown induced changes in permeability of coalbeds: a new interpretation of the reservoir response to primary recovery. Transp. Porous Media 56, 1-16 (2004)

Terzaghi, K., Peck, R.B., Mesri, G.: Soil Mechanics in Engineering Practice, 3rd edn. Wiley, New York (1996)

Thompson, M., Willis, J.R.: A reformulation of the equations of anisotropic poroelasticity. J. Appl. Mech. 58, 612-616 (1991)

Verruijt, A.: Elastic storage of aquifers. In: De Wiest, R.J.M. (ed.) Flow Through Porous Media, pp. 331-376. Academic Press, New York (1969)

Zimmerman, R.W.: Compressibility of Sandstones. Elsevier, Amsterdam (1991)

Zimmerman, R.W.: Coupling in poroelasticity and thermoelasticity. Int. J. Rock Mech. Min. Sci. 37, 79-87 (2000a)

Zimmerman, R.W. Pore compressibility under uniaxial strain. In: Carbognin, L., Gambolati, G., Johnson A.I. (eds.) Proceedings of 6th International Symposium Land Subsidence, pp. 57-65. National Research Council of Italy, Venice (2000b)

Zimmerman, R.W., Myer, L.R., Cook, N.G.W.: Grain and void compression in fractured and porous rocks. Int. J. Rock Mech. Min. Sci. 31, 179-184 (1994)

Zimmerman, R.W., Somerton, W.H., King, M.S.: Compressibility of porous rocks. J. Geophys. Res. 91, $12765-12778$ (1986) 